\title{
Comparison of the Anterior Pelvic Tilting Angle during Forward Bending in Individuals with and without Sciatic Nerve Tension
}

\author{
Kyu-hwan Choi, PT, Ph.D \\ Department of Physical Therapy, Ansan University, Ansan, South Korea
}

Background Lumbopelvic rhythm can be affected by several factors, such as stiffness of the hip joint, hamstring, and ankle, and sciatic nerve tension. Currently, no reports indicating that sciatic nerve tension affects the anterior pelvic tilting angle during forward bending are available.

Purpose This study aimed to compare the anterior pelvic tilting angle during forward bending in subjects with and without sciatic nerve tension.

Study design Cross-sectional comparative study.

Methods Eighty-eight subjects were screened and classified into two groups, namely subjects with (12 subjects) and without (12 subjects) sciatic nerve tension. Sciatic nerve tension was measured by the knee extension angle recorded in the seated position with ankle dorsiflexion using the Smart KEMA motion sensor. The mean \pm standard deviation of the knee extension angle with ankle dorsiflexion in our study population was $66.8 \pm 11.8^{\circ}$. The standard deviation was used to define the group with sciatic nerve tension $\left(<55^{\circ}\right)$ and without sciatic nerve tension $\left(>79^{\circ}\right)$. The Smart KEMA motion sensor was placed on the second spinous process of the sacrum to measure the anterior pelvic tilting angle during forward bending.

Results The mean values of the knee extension angle with ankle dorsiflexion were $46.6^{\circ}$ and $82.9^{\circ}$ for the groups with and without sciatic nerve tension, respectively. The mean values of the anterior pelvic tilting angle measured during the forward bending test were $36.7^{\circ}$ and $46.5^{\circ}$ in the groups with and without sciatic nerve tension, respectively. There was a statistically significant difference in the anterior pelvic tilting angle between the groups with and without sciatic nerve tension $(p<0.05)$.

Conclusions The results of this study suggest that sciatic nerve tension can affect the anterior pelvic tilting angle during forward bending. Further studies are needed to determine whether exercise for decreasing sciatic nerve tension can increase the anterior pelvic tilting angle during forward bending.

Key words Forward bending; Pelvic anterior tilting angle; Sciatic nerve tension; Smart KEMA.

\section{INTRODUCTION}

Lumbopelvic motion has been used frequently to identify movement impairment of the lumbar spine during forward bending. ${ }^{1,2}$ Forward bending motion is accomplished by flexion of the lumbar spine and hips. ${ }^{3}$ Several studies have reported an association between frequent forward bending
JMST

2019; 3(2): 44-48

Published Online

Dec 31, 2019

pISSN 2635-8573

eISSN 2635-8581

Article History

Received 1 Oct 2019

Revised 1 Oct 2019

(1st)

Accepted 17 Oct 2019

\section{CONTACT}

khchoi@ansan.ac.kr

Kyu-hwan Choi, Department of Physical

Therapy, Ansan

University, Ansan,

South Korea

\section{This is an Open-Access article distributed under the terms of the Creative Commons Att- ribution Non-Commercial Li- cense (http://creativecommons org/licenses/by-nc/4.0) which permits unrestricted non-co- mmercial use, distribution, and reproduction in any me- dium, provided the original work is properly cited.}

and low back pain. ${ }^{4-10}$ An activity or occupation that requires repetitive forward bending may be associated with herniated intervertebral discs in the lumbar spine. ${ }^{11}$

The individual complains of flexion-related symptoms during forward bending. Comerford and Mottram stated that an individual should be able to perform a $50^{\circ}$ forward lean of the trunk with independent hip flexion during a trunk 
lean test in the absence of uncontrolled movement. ${ }^{1}$ Esola et al. reported that subjects with a history of low back pain display greater movement in the lumbar spine than at the hips during early forward bending. ${ }^{12}$ Sahrmann proposed the concept of relative flexibility, in which the least stiff segment moves more readily than stiffer segments and the reduced length and/or stiffness of the hamstring and gluteus maximus muscles may contribute to making the lumbar spine more flexible, displaying greater flexion than the hips. $^{2}$ Excessive lumbar flexion contributes to increased loads on the low back during forward bending. ${ }^{12}$ Excessive lumbar mobility may cause tissue overload, microtrauma, and, ultimately, the development of degenerative joint and disc disease. ${ }^{2}$

Several researchers reported on the contribution of the lumbar spine and hips to forward bending, however, normative data are still limited and controversial. Burton et al. reported that a mean lumbar flexion angle of $23.9^{\circ}$ was obtained during forward bending in healthy subjects using a flexicurve measurement. ${ }^{13}$ Mayer et al. reported a mean lumbar spine motion of $55^{\circ}$ and a mean hip motion during forward bending of $42^{\circ}$ using the 2 inclinometer measurements. ${ }^{14}$ Meanwhile, Esola et al. reported a mean lumbar motion of $41.6^{\circ}$ and mean hip motion of $69.4^{\circ}$ during forward bending using a three-dimensional optoelectric motion analysis system. ${ }^{12}$

Lumbopelvic motion can be affected by several factors. Hamstring and gluteus maximus rigidity, as well as ankle and hip joint limitation of motion have been proposed as contributing factors to lumbopelvic motion during forward bending. ${ }^{2,7}$ Also, clinical observations have suggested that sciatic nerve tension may influence the lumbopelvic motion during forward bending. Therefore, we conducted our study to compare anterior pelvic tilt angle during forward bending in individuals with and without sciatic nerve tension. It was hypothesized that subjects with sciatic nerve tension would display a reduced anterior tilting angle during forward bending compared with individuals without sciatic nerve tension.

\section{METHODS}

\section{Participants}

Students from a university volunteered for this study. All participants were informed about the experimental procedures and voluntary consent was obtained. Eighty-eight subjects volunteered and were screened to classify into two groups, namely, those with sciatic nerve tension (12 subjects; 9 males and 3 females) and those without (12 subjects; 9 females and 3 males) sciatic nerve tension. For the group with sciatic nerve tension, the inclusion criterion was the presence of sciatic nerve tension while in the group without sciatic nerve tension, the inclusion criterion was absence of sciatic nerve tension in the sciatic nerve tension test. Subjects were excluded from this study if they experienced any low back, lower extremity pain, pelvic or radicular pain, or if they had a history of fracture or surgery or reduced hamstring muscle length.

\section{Procedures}

\section{1) Sciatic nerve tension test}

Sciatic nerve tension was measured using the Smart KEMA motion sensor (KOREATECH co. Ltd., Seoul, Korea). The subjects sat on the treatment table and performed hip and knee flexion creating a $90^{\circ}$ angle. The motion sensor was calibrated in this position as $0^{\circ}$ from the knee joint. The pelvis was placed in an upright position by the examiner. The examiner palpated the anterior and posterior superior iliac spine and adjusted the pelvic position at the horizontal line between the anterior and posterior superior iliac spine.

The Smart KEMA motion sensor was attached at the lateral side of the distal leg of the patient. The examiner monitored and manually stabilized the position of the tested femur and pelvis. The participants were asked to extend the tested leg with and without ankle dorsiflexion. To identify the participants with reduced hamstring length, the hamstring length test was performed. Subjects who demonstrated less than $70^{\circ}$ of the knee extension angle in the ankle plantar flexion position were excluded from this study. ${ }^{15}$ The sciatic tension test was performed on participants without reduced hamstring length. The participants were instructed to extend their leg with dorsiflexion and hold for 5 seconds. Data from the Smart KEMA motion sensor were recorded at a $25-\mathrm{Hz}$ sampling rate and transmitted to a tablet by Bluetooth to measure the knee extension angle using Smart KEMA software. Three trials were conducted for each leg and the mean of the 3 trials were used for data analysis. A 2minute resting period was provided between trials to minimize the testing effects. The leg that demonstrated greater sciatic tension was selected as the test leg. If the participant demonstrated equal sciatic nerve tension on both sides, the dominant leg was selected as the test leg.

\section{2) Anterior pelvic tilting angle during forward bending}

To measure the anterior pelvic tilting angle, the Smart KEMA motion sensor was attached to the second spinous process of the sacrum with the patient in the standing position. The motion sensor was calibrated to $0^{\circ}$ in the 
standing position. The subject was then asked to stand with both feet shoulder width apart and bend forward to touch their fingertip to a predetermined midpoint between the knee joint and ankle joint for a period of 5 seconds and was asked to maintain the position for a period of 3 seconds. The bending speed was controlled by a metronome. The participant was instructed to refrain from flexing the knee joints during the forward bending. motion Two trials were performed and the mean of the 2 trials was used for data analysis.

\section{Statistical analysis}

The Kolmogrov-Smirnov test confirmed a normal data distribution. A grouped t-test was applied to compare the anterior pelvic tilting angle between our 2 study groups (with and without sciatic nerve tension). All statistical analyses were performed by SPSS software (ver. 21.0: IBM Corp., Armonk, New York, USA). The statistical significance level was set at $\mathrm{p}<0.05$.

\section{RESULTS}

No statistically significant differences existed between our 2 study groups regarding age, height and weight ( $p>0.05$ ) (Table 1). Mean \pm standard deviation of the knee extension angle with ankle dorsiflexion of 88 subjects was $66.8 \pm 11.8^{\circ}$. The standard deviation was used to determine inclusion of the individuals in our sample in one of 2 groups: the group of individuals presenting with sciatic nerve tension (less than $55^{\circ}$ knee extension angle with ankle dorsiflexion) and the group of individuals without sciatic nerve tension (greater than $79^{\circ}$ knee extension angle with ankle dorsiflexion). The mean \pm standard deviation value of the knee extension angle with ankle dorsiflexion in the group with and without sciatic nerve tension was $46.64 \pm$ 6.77 and $82.93 \pm 3.08^{\circ}$, respectively. There was a statistically significant difference in the knee extension angle with ankle dorsiflexion between the groups in our sample $(p<0.05)$ (Table 2). The mean \pm standard deviation value of the anterior pelvic tilting angle during forward bending in the group with and without sciatic nerve tension was $36.71 \pm 10.35$ and $46.49 \pm 10.25^{\circ}$, respectively. There was a statistically significant difference in the anterior pelvic tilting angle during forward bending between our 2 study groups $(p<0.05)$ (Table 2).

\section{DISCUSSION}

The aim of our study was to compare the anterior pelvic tilting angle during forward bending between subjects with and without sciatic nerve tension. Consistent with our hypothesis, the result of the present study showed a statistically significant reduction of the anterior pelvic tilting angle during forward bending in participants with sciatic nerve tension compared with those participants without sciatic nerve tension.

Esola et al. reported that the mean angle of total forward bending in all subjects ( 20 with a history of low back pain and 21 without a history of low back pain) was $111^{\circ}$ (41.6 from the lumbar spine and $69.4^{\circ}$ from the hips). ${ }^{12}$ Reis and Macedo found that the mean pelvic tilting angle during forward bending measured by an inclinometer was $66.7^{\circ}$ in asymptomatic patients and $57^{\circ}$ in patients with chronic low back pain. ${ }^{7}$ Mayer et al. reported that the mean angle of $55^{\circ}$

Table 1. General characteristics of subjects

\begin{tabular}{ccccc}
\hline Characteristic & With SNT $(\mathrm{n}=12)$ & Without SNT $(\mathrm{n}=12)$ & $t$ & $p$ \\
\hline Age $(\mathrm{yrs})$ & $19.83 \pm 0.39$ & $19.83 \pm 0.39$ & 0.00 & 1.00 \\
Height $(\mathrm{cm})$ & $171.83 \pm 8.66$ & $165.92 \pm 8.45$ & 1.69 & 0.10 \\
Weight $(\mathrm{kg})$ & $61.83 \pm 11.00$ & $61.42 \pm 15.02$ & 0.08 & 0.94 \\
Male/female & $9 / 3$ & $3 / 9$ & N/A & N/A \\
\hline
\end{tabular}

Data are expressed as Mean $\pm \mathrm{SD} ;{ }^{*} \mathrm{SNT}$, sciatic nerve tension.

Table 2. Comparison of sciatic nerve length and forward bending between two groups

\begin{tabular}{ccccc}
\hline & With SNT $(\mathrm{n}=12)$ & Without SNT $(\mathrm{n}=12)$ & $\mathrm{t}$ & $p$ \\
\hline Knee extension angle with ankle dorsiflexion $\left(^{\circ}\right)$ & $46.64 \pm 6.77$ & $82.93 \pm 3.08$ & -16.908 & $<.01^{*}$ \\
Forward bending $\left(^{\circ}\right)$ & $36.71 \pm 10.35$ & $46.49 \pm 10.25$ & -2.327 & $.03 *$ \\
\hline
\end{tabular}

Data are expressed as Mean $\pm \mathrm{SD} ;{ }^{*} \mathrm{SNT}$, sciatic nerve tension; ${ }^{*} p<0.05$. 
for lumbar spine motion and $42^{\circ}$ for hip motion occurred during forward bending as measured by two-inclinometers. In that study, an inclinometer was placed on the posterior aspect of the sacrum, where it measured angular change in hip flexion during forward bending, which is similar to the method employed in present study. ${ }^{14}$ In the present study, the Smart KEMA motion sensor was attached on the second spinous process of the sacrum to measure the anterior pelvic tilting angle, which was $36.71^{\circ}$ in participants with sciatic nerve tension and $46.49^{\circ}$ in participants without sciatic nerve tension. The mean of the anterior pelvic tilting angle in both groups was $41.6^{\circ}$, and this mean value is consistent with the results in the Mayer et al. study. ${ }^{14}$ However, angular change of the pelvis can be affected by both femur and ankle motion; therefore, the result of the present study cannot be compared directly with the results of other studies that use methods including a photo and video-based motion analysis system.

The main finding of the present study was that participants with sciatic nerve tension demonstrated a reduced anterior pelvic tilting angle during forward bending compared with those without sciatic nerve tension. The results of this study demonstrate that sciatic nerve tension can significantly influence the anterior pelvic tilting angle during forward bending. The sciatic nerve, which is under the piriformis muscle, crosses the hip joint posteriorly; therefore, if sciatic nerve tension is increased during forward bending, discomfort or pain can result. Consequently, discomfort or pain may restrict the anterior pelvic tilting during forward bending. A decreased anterior pelvic tilting angle due to sciatic nerve tension can lead to greater lumbar flexion during forward bending. Patients with low back pain showed higher restriction in anterior pelvic tilting and greater mobility in the lumbar spine during forward bending activities. Further studies are needed to determine whether sciatic nerve tension is a risk factor for low back pain.

Hamstring tightness can affect lumbopelvic rhythm and the pelvic angle during forward bending. ${ }^{15,2,7}$ Reis and Macedo reported that hamstring tightness showed a moderate correlation with the pelvic tilting angle $(r=0.41$ for right, $r=0.44$ for left). ${ }^{7}$ In the present study, participants who had reduced hamstring length $\left(<70^{\circ}\right.$ of the knee extension angle in the ankle plantar flexion position) were excluded for a minimized hamstring extensibility effect on the anterior pelvic tilting angle during forward bending. Also, forward bending amplitude was controlled by having participants touch their fingertip to a predetermined midpoint between the knee joint and the ankle joint, rather than bend forward to the end of maximal active range, to minimize hamstring tension effect and standardize the participant's height. Tsang et al. reported that bending speed imposes different levels of demand on the kinematics and pattern of the lumbo-pelvic movement and requires the ability to regulate the lumbo-pelvic movement pattern during forward bending while standing and demonstrated in pain free individuals. ${ }^{9}$ Therefore, in the present study, bending speed was controlled by limiting execution to 5 seconds during forward bending by using a metronome.

Kim et al. reported that the lumbopelvic rhythm differed according to the low back pain subtypes, and the flexion angle of the lumbar spine was greater in the group with lumbar flexion with rotation syndrome associated low back pain than in the control group and the group with lumbar extension rotation syndrome associated low back pain; the hip flexion angle was greater in the lumbar extension rotation syndrome associated low back pain subgroup than in the control and lumbar flexion rotation syndrome group. ${ }^{16}$ In this study, the subjects were not considered if they demonstrated a lumbar movement pattern. Further studies are needed to determine whether sciatic nerve tension influences lumbopelvic rhythm differently based on a lumbar movement pattern.

It is important to note the limitations of this study. First, gender differences were found between our sample groups: 9 males and 3 females with sciatic nerve tension and 9 females and 3 males without sciatic nerve tension. Although the performed tasks were different from the present study (reaching test versus forward bending) and the sample size was smaller ( 5 men, 5 women), the Thomas et al. study reported that flexion in men occurs equally around the hips and spine, whereas flexion in women occurs primarily around the hips and knees, with minimal flexion around the spine during the reaching test. ${ }^{17}$ Differences in gender distribution between the 2 groups may affect the anterior pelvic tilting angle during forward bending. Additional studies must be conducted to determine whether there is a difference in the anterior pelvic tilting angle between males and females. This study was a cross-sectional study; therefore, it was impossible to establish a case -effect relation between sciatic nerve tension and anterior pelvic tilting angle during forward bending. We did not measure the lumbar and thoracic angle during forward bending. Therefore, it is impossible to confirm whether reduction of the anterior pelvic tilting angle causes an increased flexion angle of the lumbar or thoracic spine. Further studies are needed to measure the lumbar and thoracic sagittal angle in individuals with sciatic nerve tension to understand the lumbar and thoracic compensatory increased motion for reduction of the anterior pelvic tilting angle during forward bending. In addition, further study would be required to 
evaluate the amount of backward deviation of pelvis, because amount of backward deviation may influence anterior pelvic tilt angle during forward bending. ${ }^{2}$

\section{CONCLUSION}

The results of this study showed that the anterior pelvic tilting angle during forward bending was significantly decreased in individuals with sciatic nerve tension compared to that in individuals without sciatic nerve tension. These data suggest that interventions for decreasing sciatic nerve tension, such as nerve mobilization techniques, may be useful to restore the anterior pelvic tilting angle during forward bending activities. Further studies are needed to determine whether decrease in sciatic nerve tension is effective for increasing the anterior pelvic tilting angle during forward bending in individuals with sciatic nerve tension.

\section{Key Points}

Question Is there any difference in the anterior pelvic tilting angle during forward bending between individuals with and without sciatic nerve tension?

Findings The anterior pelvic tilting angle during forward bending in individuals with sciatic nerve tension was significantly decreased compared to that in individuals without sciatic nerve tension.

Meaning Sciatic nerve tension may affect the anterior pelvic tilting angle during forward bending; therefore, interventions for decreasing the sciatic nerve tension can be recommended for improving the anterior pelvic tilting angle during forward bending in individuals with sciatic nerve tension.

\section{Article information}

Conflict of Interest Disclosures: None.

Funding/Support: None.

Acknowledgment: None.

Ethic Approval: None.

\section{REFERENCES}

1. Comerford M, Mottram S. Kinetic control: The management of uncontrolled movement. St Louis: Churchill Livingstone; 2012.

2. Sahrmann SA. Diagnosis and treatment of movement impairment syndrome. St Louis: Mosby; 2002.

3. Nordin M, Frankel VH. Basic biomechanics of the musculoskeletal system. Philadelphia: Lea \& Febiger; 1989.
4. Magora A. Investigation of the relation between low back pain and occupation. IV. Physical requirements: bending, rotation, reaching and sudden maximal effort. Scand $J$ Rehabil Med. 1973;5(4):186-190.

5. Mellin G. Chronic low back pain in men 54-63 years of age. Correlations of physical measurements with degree of trouble and progress after treatment. Spine. 1986; 11(5):421-426.

6. Nachemson A. Disc pressure measurement. Spine. 1981; 6(1):93-97.

7. Reis FJJ, Macedo AR. Influence of hamstring tightness in pelvic, lumbar and trunk range of motion in low back pain and asymptomatic volunteers during forward bending. Asian Spine J. 2015; 9(4):535-540.

8. Svensson HO, Andersosson GB. The relationship of low back pain, work history, work environment and stress: a retrospective cross-sectional study of 38-64 years old females. Spine. 1989;14(5):517-522.

9. Tsang SMH, Szeto GPY, Li LMK, et al. The effects of bending speed on the lumbo-pelvic kinematics and movement pattern during forward bending in people with and without low back pain. BMC Musculoskelet Disord. 2017;18(1):157-168.

10. Videman T, Nurminen T, Tola S, et al. Low back pain in nurses and some loading factors of work. Spine. 1989; 14:400-404.

11. Saunders HD. Evaluation, treatment and prevention of musculoskeletal disorders. Mineapolis; Viking Press: 1985.

12. Esola MA, McClure PW, Fitzgerald GK, et al. Analysis of lumbar spine and hip motion during forward bending in subjects with and without a history of low back pain. Spine. 1996;21(1):1-8.

13. Burton AK, Tillotson KM, Troup JD. Variation in lumbar sagittal mobility with low back trouble. Spine. 1989; 14(6):584-590.

14. Mayer TG, Tencer AF, Kristofersson S, et al. Use of noninvasive techniques for quantification of spinae range of motion in normal subjects and chronic low back dysfunction patients. Spine. 1984; 9(6):588-595.

15. Lopez-Minarro PA, Alacid F. Influence of hamstring muscle extensibility on spinal curvatures in young athletes. Sci Sport. 2010; 25(4):188-193.

16. Kim $\mathrm{MH}$, Yi $\mathrm{CH}$, Kwon OY, et al. Comparison of lumbopelvic rhythm and flexion-relaxation response between 2 different low back pain subtypes. Spine. 2013;38(15):1260-1267.

17. Thomas JS, Corcos DM, Hasaan Z. The influence of gender on spine, hip, knee, and ankle motions during a reaching task. J Mot Behav. 1998; 30(2):98-103. 